\title{
A Level-Set Based \\ Volumetric CT Segmentation Technique: A Case Study with Pulmonary Air Bubbles
}

\author{
José Silvestre Silva ${ }^{1,2}$, Beatriz Sousa Santos ${ }^{1,3}$, \\ Augusto Silva ${ }^{1,3}$, and Joaquim Madeira ${ }^{1,3}$ \\ ${ }^{1}$ Departamento de Electrónica e Telecomunicações, \\ Universidade de Aveiro, Campo Universitário de Santiago, \\ P-3810-193 Aveiro, Portugal \\ \{bss, asilva, jmadeira\}@det.ua.pt \\ 2 Departamento de Física, Faculdade de Ciências e Tecnologia, \\ Universidade de Coimbra, Rua Larga, P-3004 516 Coimbra \\ jsilva@ci.uc.pt \\ ${ }^{3}$ Instituto de Engenharia Electrónica e Telemática de Aveiro \\ Campo Universitário de Santiago,P-3810-193 Aveiro, Portugal
}

\begin{abstract}
The identification of pulmonary air bubbles might play a significant role for medical diagnosis of pulmonary pathologies. A method to segment these abnormal pulmonary regions on volumetric data, using a deformable model deforming towards the objects of interest is presented. We propose a variant to the well known level-set method that keeps the level-set function moving along desired directions, with an improved stopping function that proved to be successful, even for large time steps. A region seeking approach is used instead of the traditional edge seeking. Our method is stable, robust, and automatically handles changes in surface topology during the deformation. Experimental results for 2D and 3D high resolution computed tomography images, demonstrate its performance.
\end{abstract}

\section{Introduction}

The detection of structures in the human body is difficult, due to their large variability in shape and complexity. Computed tomography (CT) is a medical imaging tool that allows volumetric data acquisition. With the high resolution computed tomography (HRCT) technique and, more recently, with multi-slice spiral CT, it is possible to obtain very thin slices of the thoracic region, having high resolution and contrast between lungs and near structures [1, 2]. CT, specially HRCT, has been one of the most used tools for pulmonary air bubble analysis. With HRCT, it is possible to observe small air bubbles, characterize them (number, dimensions, location), plan their treatment with or without surgery, and monitor their evolution (increase in size, appearance of new lesions, or additional abnormalities) [3-5].

An important goal in image processing is to detect object shapes in 2D or 3D. One way to achieve this, is to detect the associated boundaries using model based tech- 
niques. Such techniques can be, for instance, the classical snakes $[6,7]$ or 3D deformable surfaces [8,9], based on deforming an initial contour or surface towards the boundary of the object to be detected.

Level-set models, also known as geometric deformable models, have tremendous impact in medical imaging due to topology adaptability and fast shape detection, and provide an alternative solution that overcomes the limitations of parametric deformable models. Level-set models are based on curve evolution theory [10]: curves and surfaces evolve using only geometric measures, resulting in an evolution that is independent of the parameterization. The evolving curves and surfaces are represented implicitly as the level-set of a higher-dimensional function [11, 12].

The level-set implementation offers the advantage of easy initialization, computational efficiency, ability to capture sharp vertices, and simple extension to 3D. The convergence to the final result is relatively independent of the initialization.

In the present work, we consider a closed, nonintersecting, initial hypersurface placed outside the thoracic region. This hypersurface is then allowed to flow along its gradient field with a speed proportional to the hypersurface curvature and relevant image properties. We extend the known algorithms of geometric deformable models, with an improved stopping criterion. In order to characterize pulmonary air bubbles, we describe a new approach based on their physical characteristics, using a different initialization criterion and a new stopping function based on region seeking. The efficiency of the method is demonstrated with experiments on real images.

\section{The Level-Set Method}

The level-set model, initially proposed by Osher [10] and first applied to medical images, independently, by Malladi [11] and Caselles [12] is based on the equation:

$$
\frac{\partial \phi}{\partial t}=\left(K+k_{0}\right) P\|\nabla \phi\|
$$

where $\phi$ is the level-set function, $k_{0}$ is a constant, $K$ is the curvature of $\phi$ defined:

$$
K=\nabla \frac{\nabla \phi}{\|\nabla \phi\|}=\operatorname{div}\left(\frac{\nabla \phi}{\|\nabla \phi\|}\right)
$$

and $P$ is the stopping function, responsible for pushing the model towards image boundaries:

$$
P=\frac{1}{1+\left\|\nabla\left(G_{\sigma} * I\right)\right\|}
$$

where $I$ is the image, $G_{\sigma}$ is a smoothing Gaussian filter and $\sigma$ is the standard deviation of the Gaussian.

The stopping function $P$ in (3) only slows the evolution, thus, it is possible that the level-set does not stop at object boundaries, continuing its movement. To overcome this issue, some authors included an additional term [13]: 


$$
\frac{\partial \phi}{\partial t}=\left(\alpha K+k_{0}\right) P\|\nabla \phi\|+\beta \nabla P \cdot \nabla \phi
$$

where $\alpha$ and $\beta$ are constants; usually $\alpha=1$ e $\beta=1$ [13]. To improve the adjustment of the model to the image, a third term was added to the previous expression [12-14]:

$$
\frac{\partial \phi}{\partial t}=\left(K+k_{0}\right) P\|\nabla \phi\|+\nabla P \cdot \nabla \phi+\frac{k_{0}}{2} \mathbf{X} \nabla P\|\nabla \phi\|
$$

where $\mathbf{X}$ is a contour, obtained by $\phi(\mathbf{X})=0$. The last term adds an additional attraction force when the front (defined as $\phi=0$ ) is in the neighbourhood of boundaries. With this term, the model behaves better in synthetic images; but in real medical images the model with three terms isn't robust enough to process medical shapes, and, thus, only the first two terms should be used [13].

\subsection{The Stopping Function}

The first level-set model implementation used equation (1) with the stopping function described in equation (3), with $\mathrm{k}_{0}=1$. Some authors replace $K$ with $-K$, adjusting the $k_{0}$ value, when necessary; this change influences the direction of evolution, and the level-set model mainly expands (or shrinks) depending on the signs of $K[10,11]$. Common stopping functions are:

$$
P_{\exp }=\exp \left(-\left\|\nabla\left(G_{\sigma} * I\right)\right\|\right)
$$

or

$$
P_{m}=\frac{1}{1+\left\|\nabla\left(G_{\sigma} * I\right)\right\|^{m}}
$$

where $m$ is a positive integer.

A control factor of the stopping function is the image gradient. In equation (7), the relation between the stopping function and the gradient is inverse and depends on $m$. Some authors [15] use equation (6), while others $[12,16]$ use equation (7) with $m=1$ and/or $m=2$; nevertheless Malladi [11] refers both equations (6) and (7) with $m=1$.

\subsection{Extensions}

Suri [13] describes several extensions for geometric deformable models, which include: the deduction of geometric models from parametric deformable models, using internal and external energies/forces, as well as two coupled geometric models among other variations. Geometric models may need large computational resources with the increase in level-set dimension. Several authors describe methods such as the "Fast Marching Method" or the "Narrow Band Method" to improve the model evolution speed $[11,13,14,16]$. 


\section{The Proposed Level-Set Approach}

Our main purpose is to segment pulmonary air bubbles from HRCT images. The classical level-set method is not able to overcome problems caused by noise and irregular pulmonary structures. To overcome these obstacles, we have developed a level-set approach with a new initialization procedure and a new stopping function using a region seeking criterion, which has produced promising results.

Our approach starts by attenuating noise followed by the initialization of the levelset function. Then, the evolution of the level-set function is controlled by its position and curvature, and also by the stopping function. Finally, in post-processing, regions smaller than a given threshold are rejected.

\subsection{Pre-processing}

Noise is one of the main obstacles to any segmentation task. To attempt to reduce this problem, we start by smoothing the HRCT data with an average filter. Although noise is not totally removed, it is sufficiently attenuated to not significantly disturb the evolution of our level-set method.

We define the initial level-set function $\phi_{o}$, which is represented as an array with the same size as the original HRCT data, where each array element is computed as a function of its distance $d$ to the HRCT data center:

$$
\phi_{o}=L-d
$$

where $L$ is the maximum distance (e.g., for a $2 \mathrm{D}$ image, $L$ is equal to its half diagonal length, $\phi_{o}$ vertice elements have intensity values equal to zero and $\phi_{o}$ center elements are equal to $L$ ). With this initial function, all level-set values are positive and will be allowed to decrease with a speed proportional to the stopping function and to the level-set divergence.

\subsection{Evolution}

The $P$ stopping function defined by equations (6) or (7) is used by some authors to process synthetic images or well behaved medical images (that is, most of their regions have almost uniform intensity). In our case, HRCT thoracic images, specially in pulmonary regions, comprise several non uniform intensity regions. We recall that lungs are one of the organs with larger CT window Hounsfield values [17]. To surpass this problem, we have defined a new $P$ function:

$$
P=\log \left(\left|\frac{I-\varepsilon}{\gamma}\right|+1\right)
$$

where $I$ is the image, $\varepsilon$ and $\gamma$ (computed in the region to be segmented) are the mean intensity value and the dynamic range, respectively. For air bubble segmentation we used $\varepsilon=-900 \mathrm{HU}$ and $\gamma=200 \mathrm{HU}$. 
The logarithmic variation used in equation (9) has advantages when compared to a linear variation. For low $|I-\varepsilon|$ values, $P$ is small and its derivative is high, meaning that the level set is near the region to be segmented and must reduce its evolution speed (low $P$ values) to be able to detect the region to be segmented. For high $|I-\varepsilon|$ values, $P$ is large having almost null derivative, meaning that the level set is far away from the region to be segmented; moreover the evolution speed remains high and almost constant, continuing the search for regions to be segmented. Using this stopping function, the level-set will tend to adjust itself to the low intensity regions to be segmented, additional terms to impose convergence or fast evolution with constant direction (always increasing or decreasing values of $\phi$ ) no longer being needed.

From (1) we obtain the following evolution equation:

$$
\phi_{i+1}=\phi_{i}+\Delta t\left(\alpha K+k_{0}\right) P\|\nabla \phi\|
$$

that proved to be robust and of fast convergence (using $\Delta t$ values up to 10 and even higher), as long as abnormal high $\|\nabla \phi\|$ values were clipped to reasonable values.

\subsection{Post-processing}

Often, the level-set identifies not only the correct region, but also additional small regions, due to noise or irregular image texture. These unwanted small regions are discarded if their areas (or volumes for 3D data) are lower than a threshold. By definition, bubbles have a diameter no less than 1cm; to segment them in HRCT thoracic data, we reject all regions with lower size.

\section{Results}

The first experiments using our level set approach were performed on the fish cells images shown in figure 1a), using the stopping function of equation (9).

We processed three different fish cells images. Convergence was achieved after 5 to 20 iterations, depending on the image under processing, with computation time of less than a minute per image. All processing was done on a Pentium 4 computer, 1.6GHz and 256Mb of RAM, using Matlab 6.5.

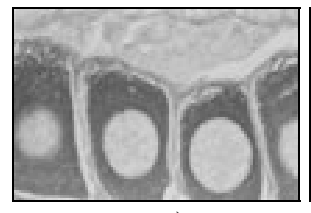

a)

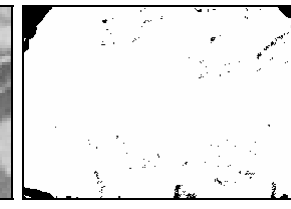

b)

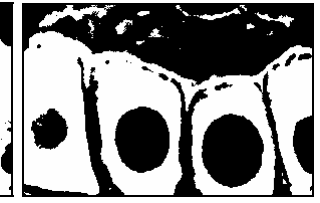

c)

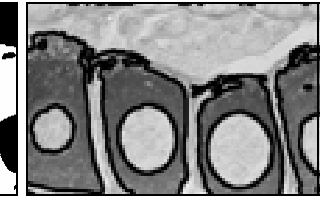

d)

Fig. 1. Fish cells images: a) original image, b) and c) are the result after 9 and 12 iterations; d) final contours overlaid on the original image. 
Our main goal is to segment pulmonary air bubbles from HRCT images. Processing one 2D image with the method described, we have successfully identified these bubbles in less than one minute, for an image of $512 \times 512$ pixels.

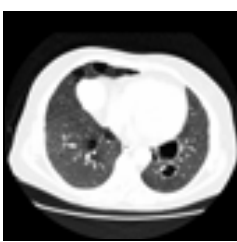

a)

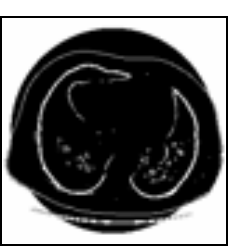

b)

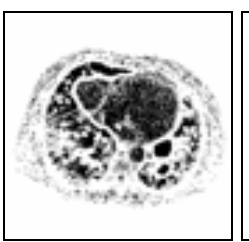

c)

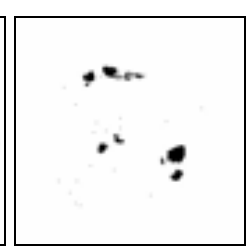

d)

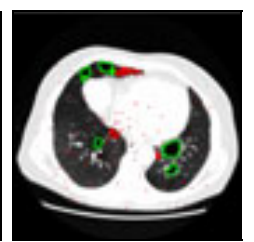

e)

Fig. 2. Thoracic HRCT image with large air bubbles: a) original image, b) through d) are the result after 2, 3 and 9 iterations; e) final contours overlaid on the original image: large contour corresponds to pulmonary air bubbles, small contours correspond to false candidates.

Applying the described method on a volume data set from a 3D HRCT exam, we are able to segment any number of pulmonary air bubbles placed anywhere inside the thoracic region. In figure 3, several air bubbles were segmented with only 5 iterations.

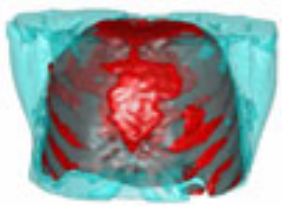

a)

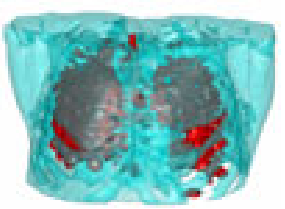

b)

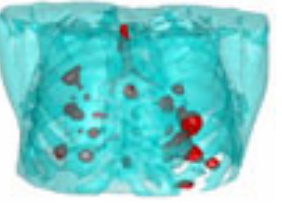

c)

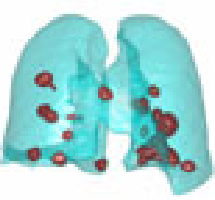

d)

Fig. 3. Image from 3D thoracic exam: a) through c) result after 1, 2 and 4 iterations; d) final image after post-processing.

\subsection{Evaluation}

To evaluate the performance of the proposed method, we used a real CT exam and inserted artificial air bubbles inside the lungs. Starting from one multi-slice CT acquisition, three exams were reconstructed from the same thoracic region, with different longitudinal resolutions $(10 \mathrm{~mm}, 5 \mathrm{~mm}, 2.5 \mathrm{~mm})$ and different number of slices (12 slices, 17 slices and 33 slices, respectively, each slice with $512 \times 512$ pixels). In each CT exam, several artificial air bubbles were inserted, with radius between $10 \mathrm{~mm}$ and $30 \mathrm{~mm}$. The processing time is proportional to the number of slices and varied from $3 \mathrm{~min}$ up to $12 \mathrm{~min}$ for exams with 10 and 33 slices, respectively. 


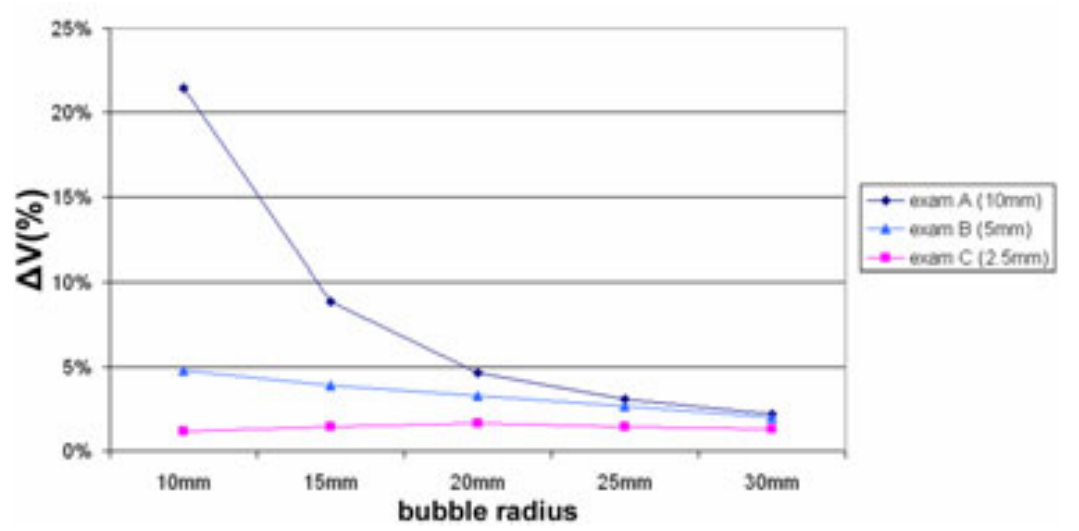

Fig. 4. Relative volume variation, for three CT exams with air bubbles of several sizes.

From figure 4, we observe that the volume error is inversely proportional to the bubble volume, as expected, and to the number of slices: the larger the bubble, the higher the method accuracy, since small bubbles have a relative high longitudinal error, due to the large difference between axial (XX, YY) and longitudinal (ZZ) resolutions.

\section{Conclusions}

We presented a new approach to segment pulmonary air bubbles. While maintaining the advantages of the traditional level-set, such as the capability of topologic transformations and working with any number of dimensions, our level-set approach, which includes a new stopping function, allows a fast convergence even for large time steps.

Motivated by the fact that HRCT thoracic images have significant noise, we developed a stopping function using a region seeking approach instead of the traditional edge seeking approach.

To overcome manual initialization, we defined and implemented an automatic initialization procedure that surrounds the complete image (2D or 3D) and doesn't depend on the objects to be segmented.

Experiments with different kinds of images were presented, which demonstrate the ability to detect several objects, as well as the power to simultaneously detect the interior and exterior cell boundaries. This approach was successfully applied in the 2D segmentation of cytoplasm fish cells and also in the segmentation of pulmonary air bubbles both in 2D and 3D HRCT images.

Although this level-set approach was developed to identify air bubbles in the lungs where the pulmonary tissues have non uniform textures due to aerial and blood trees, we believe that this segmentation method has potential applications in other medical image analysis domains, specially in 3D. The proposed method is valid for any number of dimensions. Although it was implemented in 2D and 3D, it can be applied in $\mathrm{n}-\mathrm{D}$, even in non medical imaging situations. 
Future directions for this work include the quantitative evaluation of pulmonary air bubbles by expert radiologists, as well as air-way segmentation on 3D CT exams with very thin and adjacent slices.

\section{References}

1. Brink, J., et al.: Helical CT: Principles and Technical Considerations. RadioGraphics (1994) 14:887 - 893.

2. Wang, G., P. C. Cheng, M. W. Vannier: Spiral CT: Current Status and Future Directions. Proc. SPIE (1997) 3149:203-212.

3. Morgan, M. D. L., C. W. Edwards, J. Morris, H. R. Mattews: Origin and behaviour of emphysematous bullae. Thorax (1989) 44:533-538.

4. Reid, L.: The pathology of emphysema. London: Lloyd Luke (1967).

5. Silva, J. S., A. Silva, B. S. Santos, J. Madeira: Detection and 3D representation of pulmonary air bubbles in HRCT volumes. SPIE Medical Imaging 2003: Physiology and Function: Methods, Systems, and Applications (2003) 5031:430-439.

6. Kass, M., A. Witkin, D. Terzopoulos: Snakes: Active Contour Models. International Journal of Computer Vision (1988) 1:321-331.

7. Blake, A., M. Isard: Active Contours: Springer Verlag London Limited (1998).

8. Montagnat, J., H. Delingette, N. Ayache: A Review of Deformable Surfaces: Topology, Geometry and Deformation. Image and Vision Computing (2001) 19:1023-1040.

9. McInerney, T., D. Terzopoulos: Deformable Models in Medical Image Analysis: A Survey. Medical Image Analysis (1996) 1:91-108.

10. Osher, S., J. A. Sethian: Fronts Propagation with Curvature Dependent Speed: Algorithms Based on Hamilton-Jacobi Formulations. Journal of Computational Physics (1988) 79:1249.

11. Malladi, R., J. A. Sethian, B. C. Vemuri: Shape Modeling with Front Propagation: A Level Set Approach. IEEE Transactions on Pattern Analysis and Machine Intelligence (1995) 17:158-175.

12. Caselles, V., R. Kimmel, G. Sapiro: Geodesic Active Contours. International Journal of Computer Vision (1997) 22:61-79.

13. Suri, J. S., K. Liu, S. Singh, S. N. Laxminarayan, X. Zeng, L. Reden: Shape Recovery Algorithms Using Level Sets in 2D/3D Medical Imagery: A State of the Art Review. IEEE Transactions on Information Technology in BioMedicine (2002) 6:8-28.

14. Kawata, Y., N. Niki, H. Ohmatsu, R. Kakinuma, K. Eguchi, R. Kaneko, N. Moriyama: Quantitative surface characterization of pulmonary nodules based on thin-section CT images. IEEE Transactions on Nuclear Science (1998) 45:1218-1222.

15. Kovacevic, D., S. Loncaric, E. Sorantin: Deformable Contour Based Method for Medical Image Segmentation. In: 21st International Conference on Information Technology Interfaces ITI'99 (1999).

16. Wang, H., B. Ghosh: Geometric Active Deformable Models in Shape Modeling. IEEE Transactions on Image Processing (2000) 9:302-308.

17. Hofer, M.: CT Teaching Manual. Stuttgart (Germany): Georg Thieme Verlag (2000). 